\title{
ROSE: radiology, obstruction, symptoms and exposure - a Delphi consensus definition of the association of COPD and bronchiectasis by the EMBARC Airways Working Group
}

\author{
Letizia Traversi $^{1,2}$, Marc Miravitlles $\mathbb{1}^{2,3}$, Miguel Angel Martinez-Garcia ${ }^{3,4}$, Michal Shteinberg $\mathbb{\mathbb { D }}^{5}$, \\ Apostolos Bossios $^{6}$, Katerina Dimakou ${ }^{7}$, Joseph Jacob $^{8,9}$, John R. Hurst $\mathbb{1}^{9}$, Pier Luigi Paggiaro ${ }^{10}$, \\ Sebastian Ferri $\mathbb{B}^{11,12}$, Georgios Hillas ${ }^{7}$, Jens Vogel-Claussen ${ }^{13}$, Sabine Dettmer ${ }^{13}$, Stefano Aliberti $\mathbb{B}^{12,14}$, \\ James D. Chalmers ${ }^{14,15}$ and Eva Polverino ${ }^{2}$
}

\begin{abstract}
${ }^{1}$ Dept of Medicine and Surgery, Respiratory Diseases, Università dell'Insubria, Varese-Como, Italy. ${ }^{2}$ Pneumology Dept, Hospital Universitari Vall d'Hebron, Vall d'Hebron Institut de Recerca, Barcelona, Spain. ${ }^{3} \mathrm{CIBER}$ de Enfermedades Respiratorias, Barcelona, Spain. ${ }^{4}$ Respiratory Dept, La Fe University and Polytechnic Hospital, Valencia, Spain. ${ }^{5}$ Pulmonology Institute and Cystic Fibrosis Center, Carmel Medical Center, and the Technion - Israel Institute of Technology, the B. Rappaport Faculty of Medicine, Haifa, Israel. ${ }^{6}$ Dept of Respiratory Medicine and Allergy, Karolinska University Hospital and Dept of Medicine, Karolinska Institutet, Stockholm, Sweden. ${ }^{7} 5$ th Respiratory Department, "SOTIRIA" Hospital for Chest diseases, Athens, Greece. ${ }^{8}$ Centre for Medical Image Computing, University College London, London, UK. ${ }^{9} \mathrm{UCL}$ Respiratory, University College London, London, UK. ${ }^{10}$ Dept of Surgery, Medicine, Molecular Biology and Critical Care, University of Pisa, Pisa, Italy. ${ }^{11}$ Personalized Medicine, Asthma and Allergy, Humanitas Research Hospital IRCCS, Rozzano, Milan, Italy. ${ }^{12}$ Dept of Biomedical Sciences, Humanitas University, Pieve Emanuele, Italy. ${ }^{13}$ Institute of Diagnostic and Interventional Radiology, German Center for Lung Research, Breath, Hannover Medical School, Hannover, Germany. ${ }^{14}$ IRCCS Humanitas Research Hospital, Rozzano, Italy. ${ }^{15}$ School of Medicine, University of Dundee, Ninewells Hospital and Medical School, Dundee, UK.
\end{abstract}

Corresponding author: Eva Polverino (eva.polverino@vhir.org)

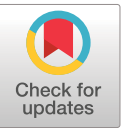

Copyright (The authors 2021

This version is distributed under the terms of the Creative Commons Attribution Non-Commercial Licence 4.0. For commercial reproduction rights and permissions contact permissions@ersnet.org

Received: 16 June 2021 Accepted: 5 Aug 2021

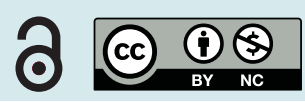

Shareable abstract (@ERSpublications)

A group of experts from EMBARC has generated a consensus definition of COPD and bronchiectasis association based on the coexistence of radiological findings, bronchial obstruction, compatible symptoms and exposure to smoke or toxic agents (ROSE criteria) https://bit.ly/3g3cdld

Cite this article as: Traversi L, Miravitlles M, Martinez-Garcia MA, et al. ROSE: radiology, obstruction, symptoms and exposure - a Delphi consensus definition of the association of COPD and bronchiectasis by the EMBARC Airways Working Group. ERJ Open Res 2021; 7: 00399-2021 [DOI: 10.1183/23120541.00399-2021].

\section{Abstract}

Introduction The coexistence of COPD and bronchiectasis seems to be common and associated with a worse prognosis than for either disease individually. However, no definition of this association exists to guide researchers and clinicians.

Methods We conducted a Delphi survey involving expert pulmonologists and radiologists from Europe, Turkey and Israel in order to define the "COPD- [bronchiectasis] BE association".

A panel of 16 experts from EMBARC selected 35 statements for the survey after reviewing scientific literature. Invited participants, selected on the basis of expertise, geographical and sex distribution, were asked to express agreement on the statements. Consensus was defined as a score of $\geqslant 6$ points (scale 0 to 9 ) in $\geqslant 70 \%$ of answers across two scoring rounds.

Results 102 (72.3\%) out of 141 invited experts participated in the first round. Their response rate in the second round was $81 \%$. The final consensus definition of "COPD-BE association" was: "The coexistence of (1) specific radiological findings (abnormal bronchial dilatation, airways visible within $1 \mathrm{~cm}$ of pleura and/or lack of tapering sign in $\geqslant 1$ pulmonary segment and in $>1$ lobe) with (2) an obstructive pattern on spirometry ([forced expiratory volume in $1 \mathrm{~s}] \mathrm{FEV}_{1} /[$ forced vital capacity] FVC <0.7), (3) at least two characteristic symptoms (cough, expectoration, dyspnoea, fatigue, frequent infections) and (4) current or past exposure to smoke ( $\geqslant 10$ pack-years) or other toxic agents (biomass, etc.)". These criteria form the acronym “ROSE” (Radiology, Obstruction, Symptoms, Exposure).

Conclusions The Delphi process formulated a European consensus definition of "COPD-BE association". We hope this definition will have broad applicability across clinical practice and research in the future. 
Introduction

COPD and bronchiectasis are two of the most frequent chronic respiratory diseases, both representing important causes of morbidity and mortality, as well as increasing burden for healthcare systems worldwide $[1,2]$. The definition of COPD is established by the Global Initiative for Obstructive Lung Disease (GOLD) as "a common, preventable and treatable disease that is characterised by persistent respiratory symptoms and airflow limitation that is due to airway and/or alveolar abnormalities usually caused by significant exposure to noxious particles or gases [3]". Recently, an international study group involving experts from Europe (EMBARC) and the USA has generated the first consensus definition of "clinically significant [bronchiectasis] BE”, including specific clinical signs/symptoms and radiological findings [4].

Although these two entities individually have very different characteristics, the presence of bronchiectasis and COPD in the same patient is quite frequent [5-7]. In fact, while usual clinical presentation of COPD is mostly driven by dyspnoea and cough [3], in bronchiectasis chronic expectoration and recurrent respiratory infections are the most frequent manifestations [8]. In the presence of compatible symptoms, the diagnostic process is also different: in COPD it is based on a functional assessment (airflow limitation after bronchodilatation), while in bronchiectasis it is based on a radiological evaluation (specific findings on computed tomography (CT) scan) [3, 8]. Additionally, risk factors are different: the exposure to smoke or other irritants is deemed necessary to confirm diagnosis of COPD, while in [bronchiectasis] BE they are less clear and extremely heterogeneous depending on the patient's age and comorbidities. Some specific conditions, such as primary immunodeficiencies [9], gastro-oesophageal reflux [10] or lung tuberculous or non-tuberculous mycobacteria infections [11], have been described as risk factors for developing bronchiectasis. Additionally, severe chronic asthma [12] or COPD [13] are also considered risk factors for developing secondary bronchiectasis. As a consequence, the epidemiology of COPD and bronchiectasis may be very different depending on risk factors and other elements such as quality of access to healthcare (spirometry and CT scan, among others) [11, 14, 15].

The epidemiology of the association between COPD and bronchiectasis is even less clear. In fact, the reported prevalence of bronchiectasis in COPD patients varies considerably across series, from $4 \%$ to $75 \%$ [5, 16-18]. Different factors can be invoked to understand such variability in prevalence rates: series differ in inclusion criteria, severity of disease, definition of bronchiectasis and method used for radiological diagnosis. Moreover, the presence of increased vessel size (such as in pulmonary hypertension), affecting the broncho-arterial ratio, could be a cause of under-diagnosis when the broncho-arterial ratio is used as diagnostic criteria on high-resolution computed tomography (HRCT) [19]. More commonly, however, bronchiectasis may be overdiagnosed, as bronchial dilatation may be found in $10-30 \%$ of CT scans of healthy and asymptomatic subjects over 40 years of age [20, 21]. Also, the presence of hypoxic vasoconstriction, frequent in chronic pulmonary diseases, could contribute to bronchiectasis overdiagnosis [17]. This variability in broncho-arterial ratio and its interpretation may influence the diagnosis of bronchiectasis and, even more frequently, in COPD patients [9].

Across the limited number of bronchiectasis studies where the prevalence of COPD has been reported, it has ranged between $8.8 \%$ and 32\% [13, 21-24]. In these studies, in the absence of an established definition of COPD-BE association, the diagnosis of COPD was based on a functional obstructive pattern and, in some cases, smoking history.

Regardless of the primary diagnosis, the coexistence of COPD and bronchiectasis is usually associated with greater symptom burden, greater frequency and severity of exacerbations, more severe airways obstruction and increased mortality risk compared with any of the two diseases alone [18, 25, 26]. Owing to all these reasons, an international group of experts has worked towards achieving a consensus definition of the association of COPD and bronchiectasis independently of the primary diagnosis. Such a definition will allow, first, a standardised epidemiological data collection and, second, to assess the health-economic burden of this association. Moreover, a consensus definition could be a critical tool to better select patients for future clinical trials.

Methods

Design of the study

This was a Delphi survey with the objective of developing an international consensus definition of the COPD-BE association.

Delphi is a consensus method developed to measure levels of agreement between experts on a subject where scientific evidence is lacking, limited or contradictory [27]. The Delphi method is based on four characteristics: 1) anonymity: guaranteed by the centralisation of questionnaire answers by a single 
moderator; 2) iteration: meaning that the same questions are reiterated in successive rounds until reaching stability in answers; 3) controlled feedback: participants are provided with feedback of previous rounds before proceeding with the following ones - the moderator can usually decide how much information will be provided after each round and the means by which this is delivered; and 4) statistical "group response", usually measured with central tendency, dispersion and frequency distributions [28-30].

Additionally, this method allows the collection of numerous expert opinions without the need of face-to-face meetings. Methods were repeatedly discussed within the panel in order to enhance validity and avoid methodological biases.

In our Delphi, a panel of experts selected the statements to be graded by survey respondents, in order to describe the association between COPD and bronchiectasis. The items receiving the highest level of concordance after two rounds were selected as the criteria forming the final definition.

\section{Participants in the survey}

The Delphi survey was designed by a panel of 16 experts in COPD and bronchiectasis from the EMBARC Airways Working Group, including a small group of radiologists with specialised interest in thoracic imaging.

The panel selected participants trying to ensure a diverse distribution by sex, geography and expertise (for COPD, bronchiectasis or both and thoracic radiology) in order to obtain a solid representation of opinions while respecting Delphi method recommendations [27-30]. We planned a target of 100 responses; therefore, assuming 20\% will be non-responders, a total of 125 invitations were sent to accredited experts in the fields of COPD, bronchiectasis and thoracic radiology from European countries, Turkey and Israel, selected based on published literature in the field. A pool of radiologists (20\% of total invitations) was included due to the important role played by radiology in the diagnosis of bronchiectasis. Moreover, the 16 members of the panel were also invited to complete the survey.

\section{Development of the survey}

Initially, the panel performed a scoping review of the literature regarding the association of COPD and bronchiectasis and discussed the findings, identifying crucial issues and common points in prior studies related to the association. After setting the aim of the definition, each panellist proposed statements for internal discussion. From the original list, the panel formulated a short list of 35 statements split into five categories: clinical, functional, microbiological, radiological and pathophysiological features (table 1).

\section{Elaboration of consensus definition}

Participants were invited to grade the selected statements from 0 (completely disagree) to 9 (fully agree). Radiologists were asked to only complete the radiology section of the survey, while clinicians completed all sections.

After the first round, the panel defined consensus as the scoring of at least 6 points (positive consensus) or fewer than 4 points (negative consensus) in $\geqslant 70 \%$ of the responders, according to Delphi methodology [21]. Furthermore, after the analysis of results from the first round, the second round was designed where some questions were reiterated, some were reformulated to improve clarity and some new questions were added to enhance the quality of the definition.

Finally, the panel formulated the consensus definition with the criteria that reached the higher level of consensus at the end of the second round.

Results

\section{First round}

The first round of the survey was open from January 30 to April 24, 2019, with 102 responders (response rate $72 \%$, females $40 \%$ ). Among them, $18 \%$ were radiologists, 38\% were COPD experts, $38 \%$ bronchiectasis experts and 7\% were pulmonologists working on both diseases. Responders represented 30 countries: Austria, Belgium, Bulgaria, Croatia, Denmark, Estonia, France, Germany, Greece, Ireland, Israel, Italy, Latvia, Lithuania, Macedonia, Malta, Moldova, the Netherlands, Norway, Poland, Portugal, Romania, Russia, Serbia, Slovenia, Spain, Sweden, Switzerland, Turkey and the UK.

Despite the initial attempt to have a homogeneous distribution of survey participants from different European regions, we finally had seven additional responders from countries with more centres dedicated to COPD and bronchiectasis in order to ensure the target number of 100 participants (figure 1). 
TABLE 1 Final list of statements included in the Delphi survey

Clinical items required to define the association of COPD and bronchiectasis

1. Age $>35$ years

2. Current or past smoking habit ( $\geqslant 10$ pack-years) or other toxic exposure (biomass, industrial, etc.)

3. Presence of at least $15 \mathrm{~mL}$ of expectorated sputum most of the days

4. Presence of purulent sputum most of the days

5. Presence of haemoptysis

6. Presence of chronic cough and expectoration for at least 3 consecutive months in the last 2 years

7. Presence of dyspnoea ( $m M R C \geqslant 1$ ) in the last 2 years

8. History of at least one exacerbation in the previous year

9. History of frequent infectious exacerbations $(\geqslant 2)$

10. History of at least one severe exacerbation in the last year (hospitalisation or intravenous antibiotic therapy)

Radiological items required to define the association of COPD and bronchiectasis

11. Lack of airway tapering sign

12. Airways visible within $1 \mathrm{~cm}$ of the pleural surface

13. $\mathrm{BE}$ (bronchial dilatation) in at least one pulmonary segment in one lobe

14. BE (bronchial dilatation) in more than one pulmonary segment in one lobe

15. $\mathrm{BE}$ (bronchial dilatation) in one or more pulmonary segments in more than one lobe

16. Presence of extensive emphysema

17. Extensive bronchial wall thickening

18. Presence of diffuse mucus plugging

19. Presence of air trapping

Functional items required to define the association of COPD and bronchiectasis

20. Post-bronchodilator $\mathrm{FEV}_{1} / \mathrm{FVC}<0.7$

21. Post-bronchodilator $\mathrm{FEV}_{1} / \mathrm{FVC}<\mathrm{LLN}$

22. A positive BD test excludes the diagnosis of COPD-BE association

23. A mixed (restrictive/obstructive) pattern excludes the diagnosis of COPD-BE association

24. A history of positive BD test excludes the diagnosis of COPD-BE overlap

25. A documented history of asthma excludes the diagnosis of COPD-BE overlap

Microbiological items required to define the association of COPD and bronchiectasis

26. At least one isolation of PPM in sputum in the last year in steady state

27. $>1$ isolation of PPM in sputum in the last year in steady state

28. History of chronic bronchial infection by any PPM in steady state

29. Isolation of Pseudomonas aeruginosa in sputum

30. No isolation of PPM

Pathophysiological items required to define the association of COPD and bronchiectasis

31. Blood leukocytosis in stable clinical conditions

32. High blood level of C-reactive protein in stable clinical conditions

33. High blood level of fibrinogen in stable clinical conditions

34. High neutrophil count in sputum in stable clinical conditions

35. High eosinophil count in serum in stable clinical conditions

Participants were asked to grade each item, according to their level of agreement on the necessity for the statement to be part of the COPD-BE association definition. mMRC: modified Medical Research Council; BE: bronchiectasis; $\mathrm{FEV}_{1}$ : forced expiratory volume in $1 \mathrm{~s}$; FVC: forced vital capacity; LLN: lower limit of normal; BD: bronchodilation; COPD: chronic obstructive pulmonary disease; PPM: potential pathogenic microorganisms.

After the analysis of results from the first round, five statements reached positive consensus and passed to the second round: "airways visible within $1 \mathrm{~cm}$ of the pleura"; "BE in at least one pulmonary segment in more than one lobe"; "lack of tapering sign"; "post-bronchodilator [forced expiratory volume in $1 \mathrm{~s}$ ] $\mathrm{FEV}_{1}$ / [forced vital capacity] FVC <0.7”; “current or past smoking habit ( $\geqslant 10$ pack-years) or history of other exposure to toxic inhalants (biomass, industrial, etc)” (figure 2).

Negative consensus was reached only by two statements: 1) "a mixed ventilatory pattern excludes the diagnosis"; and 2) "history of positive bronchodilation test excludes the association".

In the radiology section, three very similar assertions about distribution of bronchiectasis obtained a high score, although only one reached the consensus threshold. Therefore, the panel decided to include all of them in the second round in order to reiterate the information and achieve a better agreement.

All the other statements obtained an intermediate result and therefore were excluded from the second round. No microbiological, clinical or pathophysiological items passed the first round, except for history of 
Northern Europe

Central/Eastern Europe

Southern Europe ${ }^{\#}$

Western Europe
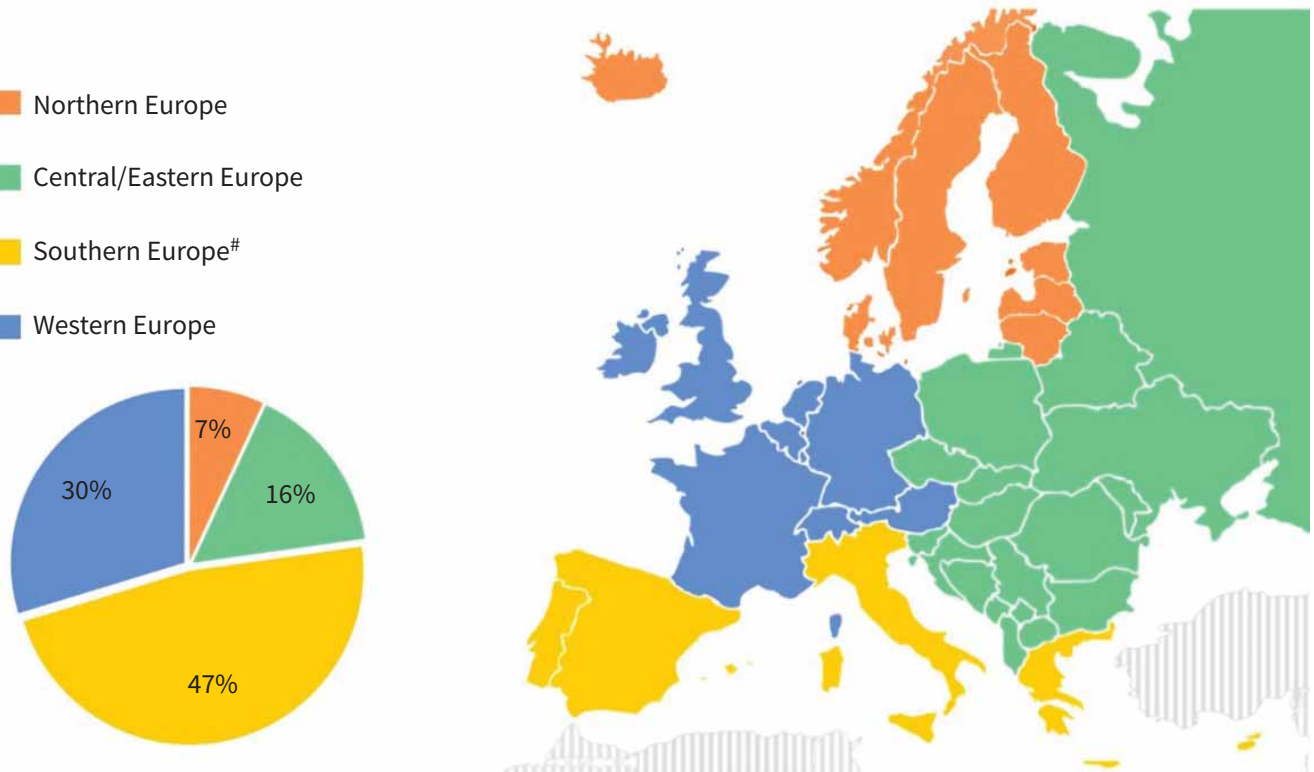

FIGURE 1 Geographical distribution of participants to the survey. Countries have been grouped according to EuroVoc criteria [24]. ": only European countries are represented in the map; responders from Turkey and Israel have been included in the "Southern Europe" region.

exposures. To confirm the exclusion of these potentially relevant categories from the definition, the panel introduced new specific questions regarding microbiological and clinical items and gave responders the opportunity to add items with two optional open questions (table 2).

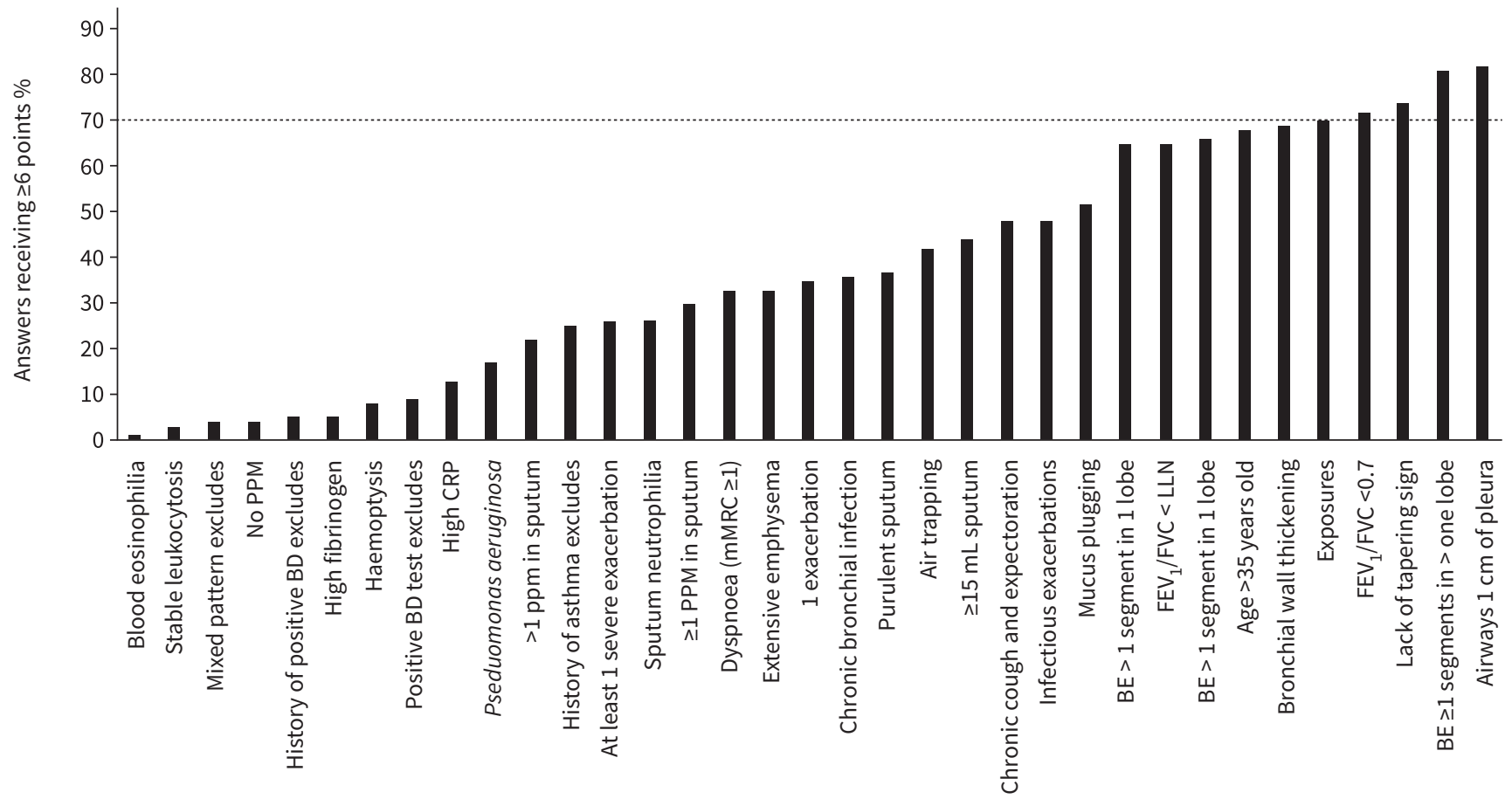

FIGURE 2 Results from the first round. Statements were graded from 1 (completely disagree) to 9 (completely agree). Consensus was defined as at least $70 \%$ of answers scored 6 or higher (dashed line). PPM: potential pathogenic microorganisms; BD: bronchodilation test; CRP: C-reactive protein; mMRC: modified Medical Research Council; BE: bronchiectasis; FEV $_{1}$ : forced expiratory volume in $1 \mathrm{~s}$; FVC: forced vital capacity; LLN: lower limit of normal. 
TABLE 2 Second round: additional questions ${ }^{\#}$

Clinical items required to define the association of COPD and bronchiectasis

In addition to the criteria already selected, should any clinical aspect be included in order to ensure only symptomatic patients are considered? (YES/NO)

If answered yes to this question, then chose one of the following:

a) Should the definition of COPD-BE include at least ONE of the following signs and symptoms: cough, expectoration, dyspnoea, fatigue, frequent infections $(\geqslant 2)$ ?

b) Should the definition of COPD-BE include at least TWO of the following signs and symptoms: cough, expectoration, dyspnoea, fatigue, frequent infections $(\geqslant 2)$ ?

c) OPEN QUESTION: is there a sign or symptom you consider essential to define COPD-BE association?

Microbiological items required to define the association of COPD and bronchiectasis

In addition to the criteria already selected, should any microbiological aspect be included? (YES/NO) If answered yes to this question, then chose one of the following:

a) To define COPD-BE association the following criterion is required: at least one isolation of PPM in sputum in the last year in steady state

b) To define COPD-BE association the following criterion is required: $>1$ isolation of PPM in sputum in the last year

c) To define COPD-BE association the following criterion is required: history of chronic bronchial infection (two or more isolates of the same organism at least 3 months apart in 1 year, see ERS guidelines) by any PPM

BE: bronchiectasis; PPM: potentially pathogenic microorganisms. "\#: in case of an affirmative answer to the primary question (in bold), secondary questions were performed.

\section{Second round}

The second round ran between the end of June and the end of July 2019 and 16 statements were included: five on clinical features, five on radiological features, one on functional features and five on microbiological features (table 3). All responders from the first round were contacted and invited to

\begin{tabular}{|c|c|c|}
\hline Confirmation of statements & $\%$ of answers & Mean score \\
\hline Smoking habit and exposures" & 85 & 7.7 \\
\hline Post-bronchodilator $\mathrm{FEV}_{1} / \mathrm{FVC}<0.7^{\#}$ & 93 & 8.1 \\
\hline Lack of tapering sign & 83 & 7.3 \\
\hline Airways $<1 \mathrm{~cm}$ of pleura" & 87 & 7.1 \\
\hline $\mathrm{BE} \geqslant 1$ pulmonary segment in one lobe & 64 & 5.8 \\
\hline $\mathrm{BE}>1$ pulmonary segment in one lobe ${ }^{q}$ & 70 & 6.2 \\
\hline $\mathrm{BE} \geqslant 1$ pulmonary segment in more than one lobe & 73 & 6.6 \\
\hline \multicolumn{3}{|l|}{ Clinical and microbiological aspects ${ }^{\#}$} \\
\hline 2.1 Should any clinical aspect be included? & YES & $76 \%$ \\
\hline Between "yes" responders $(n=51)$ & $\%$ of answers & Mean score \\
\hline At least ONE symptom & 65 & 5.9 \\
\hline At least TWO symptoms & 84 & 7.5 \\
\hline 2.2 Should any microbiological aspect be included? & YES & $39 \%$ \\
\hline Between "yes" responders $(n=26)$ : & $\%$ of answers & Mean score \\
\hline At least one isolation of PPM stability & 65 & 6.1 \\
\hline More than one isolation of PPM in sputum in the last year & 54 & 5.7 \\
\hline Chronic bronchial infection by any PPM & 65 & 6.4 \\
\hline Pseudomonas ever isolated in sputum & 46 & 5.2 \\
\hline \multicolumn{3}{|c|}{$\begin{array}{l}\text { In the second column, the percentage of answers graded } 6 \text { points or more. In the third column, the mean score } \\
\text { received. } \mathrm{FEV}_{1} \text { : forced expiratory volume in the } 1 \mathrm{~s} \text {; FVC: forced vital capacity; BE: bronchiectasis; PPM: potential } \\
\text { pathogenic microorganisms. Where not specified, expressed percentages refer to the number of responders: } \\
\text { \#: } 67 \text { responders (only pulmonologists); }{ }^{9}: 83 \text { responders (pulmonologists+radiologists). }\end{array}$} \\
\hline
\end{tabular}


TABLE 4 Answers to open question "is there a sign or symptom you consider essential to define BE-COPD association"

\begin{tabular}{lll} 
Open question & Answers n & Detailed answers \\
\hline $\begin{array}{l}\text { Total responders } \\
\text { Expectoration }\end{array}$ & 29 & Bronchorrhea, chronic sputum, chronic phlegm, phlegm production, purulent sputum \\
\hline $\begin{array}{l}\text { Cough } \\
\text { Recurrent infections }\end{array}$ & 11 & $\begin{array}{l}\text { Frequent infections, frequent lower respiratory infections, more than one exacerbation per year, infections } \\
\text { including pneumonia }\end{array}$ \\
\hline Answers were grouped into three clusters according to similarity of answers. Detailed answers are listed in third column.
\end{tabular}

participate once again. The response rate at the second round was $81 \%$ (83 responders). As for the first round, radiologists (17 out of 83 ) were asked to answer exclusively the radiology section, while respiratory specialists (67 out of 83) completed the entire survey.

As expected, all the questions that reached consensus at the first round were confirmed, with a higher level of concordance between responders.

With regard to clinical features, 51 participants (76\% of the pulmonologists) agreed on the need to include at least a clinical criterion in the final definition of COPD-BE association. Among them, 84\% (43 out of 51) chose to include the presence of at least two symptoms between those listed (cough, expectoration, dyspnoea, fatigue, frequent infections) in the definition. 29 “yes” responders (56\%) also answered the open question: all the proposed symptoms were coherent with those listed in the previous list (table 4).

Conversely, the exclusion of microbiological characteristics from the definition was widely confirmed by $61 \%$ of pulmonologists (41 out of 67) (table 3). Owing to the high consensus reached, the panel decided not to perform a third round.

According to final responses of the second round, the panel formulated the consensus definition expressed in table 5.

These criteria form the "ROSE” acronym (Radiology, Obstruction, Symptoms, Exposure, figure 3).

\section{Discussion}

The need to phenotype chronic respiratory disease patients has been frequently raised, mostly in order to adopt personalised treatments and improve outcomes [31-32]. This is particularly challenging when different pathological conditions coexist, as in the case of COPD-BE association.

The presence of COPD and bronchiectasis in a single patient has frequently been reported [5-7, 17] Furthermore, numerous studies have shown that this association has a more severe clinical presentation and worse prognosis than each individual disease [17, 18, 33-38]. However, there is no consensus on how this association should be defined. Indeed, many experts have provided different interpretations of this association [5, 39]. Some authors such as Hurst et al. [5, 40] have discussed the existence of a "COPD$\mathrm{BE}$ overlap syndrome”, which might suggest the existence of a unique and distinctive clinical entity with different clinical, radiological or biological characteristics compared to the two diseases alone [5]. However, after internal discussion, the panel decided not to adopt an overlap definition for coexisting

\section{TABLE 5 Final consensus definition}

The association of COPD and bronchiectasis is defined as the presence of at least four elements

1. RADIOLOGICAL: Abnormal bronchial dilatation in one or more pulmonary segment in more than one lobe and specific radiological findings (airways visible within $1 \mathrm{~cm}$ of pleura and/or lack of tapering sign) plus

2. OBSTRUCTION: a functional obstructive pattern (post-bronchodilator $\mathrm{FEV}_{1} / \mathrm{FVC}<0.7$ ), plus

3. SYMPTOMS: two or more of the following symptoms: cough, expectoration, dyspnoea, fatigue, frequent lower airway infections ( $\geqslant 2 /$ year) plus

4. EXPOSURE: current or past smoking habit ( $\geqslant 10$ pack-years) or other toxic exposure (biomass, industrial, etc.)

$\mathrm{FEV}_{1}$ : forced expiratory volume in the $1 \mathrm{~s} ; \mathrm{FVC}$ : forced vital capacity. 


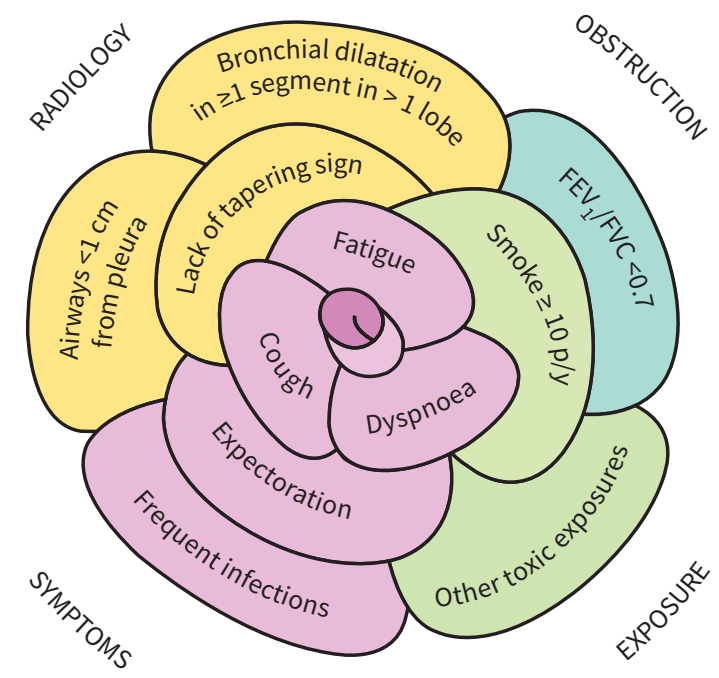

FIGURE 3 The "ROSE" criteria: Radiology, Obstruction, Symptoms, Exposure, defining the association of COPD and bronchiectasis. $\mathrm{FEV}_{1}$ : forced expiratory volume in $1 \mathrm{~s}$; FVC: forced vital capacity; $\mathrm{p} / \mathrm{y}$ : pack-years.

COPD and bronchiectasis, considering the lack of supporting evidence. At the present time, the understanding of biological and pathogenetic interaction between these two conditions is still poor and, in our opinion, definitely not enough to justify the description of a new clinical entity. Alternative concepts have been proposed, including the idea of using clinical criteria to determine the "dominant" condition for therapy, either bronchiectasis as a phenotype of COPD or chronic airflow obstruction in a patient with bronchiectasis. This approach is difficult to standardise across different centres as objective methods of defining dominant conditions have not yet been defined. Instead, the panel decided to consider the presence of COPD and bronchiectasis as an association of two different defined diseases, with complex and not fully understood mutual interactions.

The pathophysiology of this association is still uncertain. Indeed, it is not clear whether the presence of bronchiectasis could increase susceptibility to tobacco smoking, leading to more rapidly progressive functional and clinical decline and, therefore, the development of COPD; or conversely, whether the development of bronchiectasis could be part of the natural history of COPD [5]. Recently, a long-term observational study by Martinez-Garcia et al. [26] identified the presence of chronic purulent sputum production, potentially pathogenic microorganisms (PPM) in sputum and frequency of hospitalisations due to COPD exacerbations as independent, preventable and treatable risk factors for bronchiectasis emergence or progression in COPD patients. More data are needed to unravel this issue, and we believe that a definition is crucial in order to optimise the development of evidence in the field, to guide future trials and to produce useful clinical recommendations.

To satisfy these needs, the definition should be simple, clear and helpful, and ideally useful for clinical settings as well as for research. Owing to the lack of agreement in the current literature, we decided to approach this problem by assessing the opinion of professionals with different backgrounds, through a Delphi survey and, finally, to produce a consensus definition. To our knowledge, this is the first attempt to systematically address this need.

An optimal geographical distribution of responders was sought, but unfortunately it could be only partially achieved. In fact, Eastern Europe, the most populated European region, only accounted for $16 \%$ of respondents, while most came from the UK, Spain and other central and southern European countries. It possibly reflects the different levels of experience and scientific interest in bronchiectasis across European countries, also suggested by preliminary data from the European bronchiectasis registry (EMBARC) [41]. Of course, this distribution is a potential source of bias, due to the variability between different geographical areas in terms of bronchiectasis aetiologies, prevalence of COPD, smokers, tuberculosis, etc. 
The final consensus definition of the association of COPD and bronchiectasis corresponds to the acronym ROSE for the four components: Radiology, Obstruction, Symptoms and Exposure. Responses obtained from participants reflect the importance of radiology for the diagnosis of bronchiectasis in COPD patients. In fact, highest consensus was obtained by statements derived from Naidich criteria [42]; however, statements regarding the extent and distribution of bronchiectasis had to be reiterated on the second round for confirmation, when finally a clear agreement was achieved: abnormal bronchial dilatation needs to be extensive ("one or more pulmonary segment in more than one lobe") to define the pathological association of COPD and bronchiectasis.

Other radiological criteria such as the presence of bronchial wall thickening received a borderline score (68\%). Both radiologists and pulmonologists in the panel agreed on excluding it from the second round, being a nonspecific marker of inflammation present in different respiratory conditions. Similarly, the broncho-arterial ratio was not included in the statements of our survey, due to its lack of specificity for the association COPD-BE. As stated before, the broncho-arterial ratio in patients with both COPD and bronchiectasis can be influenced by several factors, such as pulmonary hypoxic vasoconstriction, advanced age and increased vessel size due to pulmonary hypertension, among others [19, 43].

According to GOLD, bronchial obstruction is needed to define the presence of COPD [3], therefore representing an essential feature in any comorbid association. Indeed, the presence of airflow obstruction is a necessary but not sufficient condition to establish a COPD diagnosis. Conversely, in pure bronchiectasis a wide variability of functional patterns has been described [23]. Persistent airflow obstruction is frequent in symptomatic bronchiectasis patients without smoking history or other risk factors for COPD. This could be associated with misdiagnosed COPD in a variable proportion of bronchiectasis patients, possibly leading to a delay in bronchiectasis diagnosis if HRCT is not performed [23, 44-46].

Regarding symptoms, no clinical criteria reached the expected level of consensus in the first round. Nevertheless, considering the importance of clinical manifestations in both COPD and bronchiectasis, the panellists considered the possibility that the first-round statements might have lacked clarity and reformulated them in the second round, getting the approval of most responders. Indeed, the included symptoms are unspecific and common to a variety of chronic respiratory conditions, but still necessary to define this clinical association. The panel used the following threshold to define "frequent lower airway": at least two exacerbations/year or one hospitalisation/year. This decision was taken in consideration of the available literature on frequent exacerbator phenotypes in both COPD and bronchiectasis [47-51]. The inclusion of smoking or toxic exposures received a high level of consensus since the first round. In fact, cigarette smoking represents the main risk factor for COPD in high-income countries [3]. The threshold of 10 pack-years was chosen since it is generally accepted as the minimum significant exposure to develop COPD [3]. Biomass exposure was also considered for the definition as it can be a relevant cause of COPD in low-income countries [52], and COPD development can be expected after 10-15 years of exposure [53, 54].

Apart from the criteria that were included in the ROSE definition, we explored other aspects of COPD-BE association that did not reach consensus. For instance, none of the statements proposed in the pathophysiological section achieved consensus, highlighting the fact that we currently lack any relevant biological marker of this association. Among the excluded variables, age $>35$ years (68\%) obtained a borderline score. In concordance with the responders, although the association of COPD-BE is a disease of the adult population, the panellists also felt that it was impossible to define an age threshold for the onset of both COPD and bronchiectasis. This decision finds confirmation in a prevalence study from China, where an increasing number of individuals with COPD were $>20$ years old [55].

Despite the importance of respiratory infections, respondents did not consider microbiological criteria essential to define the COPD-BE association. Respiratory infections are one of the most frequent clinical manifestations and alarm signs to suspect bronchiectasis, while in COPD they are a potential marker of disease severity and/or can suggest the need for a therapeutic step-up. Nevertheless, respiratory infections do not represent an exclusive criterion to define any respiratory condition, being nonspecific complications of most airway diseases.

However, in view of ongoing research on clinical and biological phenotypes of the COPD-BE association, the perceived importance of biological or microbiological markers could change in the future. For example, isolation of Pseudomonas aeruginosa or Haemophilus influenzae has already been identified as a risk factor for bronchiectasis in patients with COPD [25, 56, 57]. Moreover, both bacterial and fungal infections of the airways have been described as being more prevalent in patients with both COPD and bronchiectasis [58-58], though no specific phenotypes have been identified yet. 
In conclusion, the EMBARC Working Group on Airways Diseases agreed that the definition of COPD-BE association requires the coexistence of specific radiological signs and a functional obstructive pattern associated with the presence of at least two characteristic symptoms and current or past exposure to smoke or other toxic agents, which can be summarised in the acronym ROSE.

Future validation of this definition in a clinical setting is necessary, together with pheno- and/or endotyping studies to assess the biological and functional patterns of patients with COPD and bronchiectasis. In this group of patients, the identification of treatable traits will be important for management, appropriate enrolment in clinical trials and for new drug registration purposes.

Hopefully, the present consensus definition of COPD-BE can be the first step in guiding risk stratification and clinical management of patients with this association after an appropriate validation in international cohorts of patients with COPD and/or bronchiectasis.

Acknowledgements: We gratefully acknowledge experts who took the time to participate in our survey (in alphabetical order): Alan Altraja, pneumologist, Estonia; Adelina Amorim, pneumologist, Portugal; Alvar Agusti, pneumologist, Spain; Sergey Avdeev, pneumologist, Russia; Judith Babar, radiologist, UK; Petros Bakakos, pneumologist, Greece; Adam Barczyk, pneumologist, Poland; Miriam Barrecheguren, pneumologist, Spain; Danielle Bendayan, pneumologist, Israel; Francesco Blasi, pneumologist, Italy; Anne Laure Brun, radiologist, France; Guy Brusselle, pneumologist, Belgium; Pierre-Regis Burgel, pneumologist, France; Mathieu Buscot, pneumologist, France; Myriam Calle, pneumologist, Spain; Ciro Casanova, pneumologist, Spain; Eva Castañer, radiologist, Spain; Joanna Chorostowska-Wynimko, pneumologist, Poland; Joao Cordeiro Da Silva, pneumologist, Portugal; Nuno Cortesao, pneumologist, Portugal; Edvardas Danila, pneumologist, Lithuania; David De la Rosa, pneumologist, Spain; Sanja Dudvarski, pneumologist, Serbia; Zvi Fridlender, pneumologist, Israel; Rosa Maria Giron, pneumologist, Spain; Caroline Gouder, pneumologist, Malta; Hakan Gunen, pneumologist, Turkey; Matevz Harlander, pneumologist, Slovenia; Emilia Hardak, pneumologist, Israel; Ana Hećimović, pneumologist, Croatia; Mats Hemlin, pneumologist, Sweden; Juan Luis Rodríguez-Hermosa, pneumologist, Spain; Wim Janssens, pneumologist, Belgium; Maria Kaponi, pneumologist, Greece; Vladimir Koblizek, pneumologist, Czech; Eva Kocova, radiologist, Czech Republic; Malcolm Kohler, pneumologist, Switzterland; Konstantinos Kostikas, pneumologist, Greece; Kosta Kostov, pneumologist, Bulgaria; Rottem Kuint, pneumologist, Israel; Beatriz Lara, pneumologist, UK; Stelios Loukides, pneumologist, Greece; Beatrice Mahler, pneumologist, Romania; Luis Maiz-Carro, pneumologist, Spain; Diana Manolescu, radiologist, Romania; Alicia Marin, pneumologist, Spain; Zinka Matkovic, pneumologist, Croatia; Melissa Jane McDonnell, pneumologist, Ireland; Jordan Minov, pneumologist, Macedonia; Eduard Monsó, pneumologist, Spain; Jean-François Mornex, pneumologist, France; Oxana Munteanu, pneumologist, Moldova; Marlene Murris, pneumologist, France; Arjun Nair, pneumologist, UK; Joao Neves, pneumologist, Portugal; Mariaelena Occhipinti, radiologist, Italy; Arlette Odink, radiologist, the Netherlands; Esther Pallisa, radiologist, Spain; David Parr, pneumologist, UK; Alexander Poellinger, radiologist, Switzerland; Concepcion Prados, pneumologist, Spain; Helmut Prosch, radiologist, Austria; Dejan Radovanovic, pneumologist, Italy; Felix Ringshausen, pneumologist, Germany; Nicolas Roche, pneumologist, France; Robert Rutherford, pneumologist, Ireland; Marcelo Sanchez, radiologist, Spain; Abdulah Sayiner, pneumologist, Turkey; Sonia Schneer, pneumologist, Israel; Niels Seersholm, pneumologist, Denmark; Sarah Sheard, radiologist, UK; Trude Sildnes, radiologist, Norway; Mario Silva, radiologist, Italy; Dusan Skrbic, pneumologist, Serbia; Juan Jose Soler-Cataluña, pneumologist, Spain; Antonio Spanevello, pneumologist, Italy; Robert Stockley, pneumologist, UK; Daiana Stolz, pneumologist, Switzerland; Maria Sucena, pneumologist, Portugal; Claudio Tantucci, pneumologist, Italy; Xristina Triantaffyllidou, pneumologist, Greece; Alice Turner, pneumologist, UK; Ruxandra Ulmeanu, pneumologist, Romania; Fatma Işıl Uzel, pneumologist, Turkey; Eva Van Braeckel, pneumologist, Belgium; Tom Vegard-Macussen, radiologist, Norway; Montserrat Vendrell, pneumologist, Spain; Jorgen Vestbo, pneumologist, UK; Tom Wilkinson, pneumologist, UK.

Provenance: Submitted article, peer reviewed.

Conflict of interest: L. Traversi has nothing to disclose. M. Miravitlles reports personal fees from AstraZeneca, Boehringer Ingelheim, Chiesi, Cipla, Menarini, Rovi, Bial, Sandoz, Zambon and CSL Behring, grants and personal fees from Grifols, personal fees from Novartis, grants and personal fees from GlaxoSmithKline, and personal fees from Gebro Pharma, Kamada, Laboratorios Esteve, Ferrer, Mereo Biopharma, Verona Pharma, TEVA, Spin Therapeutics, PH Pharma and from Sanofi, outside the submitted work. M.A. Martinez-Garcia reports personal fees from Grifols, grants and personal fees from TEVA, personal fees from Novartis and Zambon, grants and personal fees from Philips and Vitalair, and personal fees from Chiesi, AZ and GSK, outside the submitted work. M. Shteinberg reports grants and personal fees from GSK and Novartis, grants from Trudell Pharma, and personal fees from Actelion, Boehringer Ingelheim, Rafa, AstraZeneca, TEVA, Kamada, Horizon Pharma and Vertex pharmaceuticals, outside the submitted work. A. Bossios reports personal fees from GlaxoSmithKline, TEVA, Novartis, Sanofi and AstraZeneca outside the submitted work. K. Dimakou reports personal fees from Chiesi, 
AstraZeneca, Pfizer, Boehringer Ingelheim, Novartis, GlaxoSmithKline and Menarini outside the submitted work. J. Jacob reports grants from Wellcome Trust Clinical Research Career Development, Roche, Boehringer Ingelheim, Novartis, GlaxoSmithKline and NHSX outside the submitted work. J.R. Hurst reports personal fees from AstraZeneca, Boehringer Ingelheim, Chiesi and Novartis outside the submitted work. P.L. Paggiaro reports personal fees from Alk-Abellò, AstraZeneca, Chiesi, GSK, Guidotti, Menarini, Mundipharma, Novartis and Sanofi outside the submitted work. S. Ferri has nothing to disclose. G. Hillas reports personal fees from AstraZeneca, Boehringer Ingelheim, Chiesi, CSL Behring, ELPEN, Innovis, GSK, Menarini, Novartis, Pharmaten and UCB outside the submitted work. J. Vogel-Claussen reports grants and personal fees from Siemens Healthineers, AstraZeneca, GSK, Novartis and Boehringer Ingelheim outside the submitted work. S. Dettmer has nothing to disclose. S. Aliberti reports personal fees from Bayer Helthcare, Grifols, AstraZeneca and Zambon, grants and personal fees from Chiesi and INSMED, personal fees from GlaxoSmithKline, Menarini and ZetaCube Srl, and grants from Fisher \& Paykel, outside the submitted work. J.D. Chalmers reports grants and personal fees from AstraZeneca, Boehringer Ingelheim, Chiesi and GlaxoSmithKline, grants from Gilead Sciences, Novartis and Insmed, personal fees from Janssen and Zambon, and grants and personal fees from Grifols, outside the submitted work. E. Polverino reports personal fees from Bayer, grants and personal fees from Grifols, and personal fees from Insmed, Chiesi, Menarini, Zembon and Pfizer, outside the submitted work.

Support statement: J. Jacob is supported by Clinical Research Career Development Fellowship 209553/Z/17/Z from the Wellcome Trust and the NIHR University College London Hospital Biomedical Research Centre. A. Bossios is supported by Swedish Heart and Lung Foundation fellowship 20180219.

\section{References}

1 Ferkol T, Schraufnagel D. The global burden of respiratory disease. Ann Am Thorac Soc 2014; 11: 404-406.

2 Goeminne PC, Hernandez F, Diel R, et al. The economic burden of bronchiectasis - known and unknown: a systematic review. BMC Pulm Med 2019; 19: 54.

3 Global Initiative for Chronic Obstructive Lung Disease (GOLD). Global Strategy for the Diagnosis, Management and Prevention of COPD. 2020. Available from: http://goldcopd.org.

4 Aliberti S, Goeminne PC, O'Donnell AE, et al. Criteria and definitions for the radiological and clinical diagnosis of bronchiectasis in adults for use in clinical trials: international consensus recommendations. Lancet Respir Med 2021; in press [http://dx.doi.org/10.1016/S2213-2600(21)00277-0].

5 Martinez-Garcia MA, Miravitlles M. Bronchiectasis in COPD patients: more than a comorbidity? Int J Chron Obstruct Pulmon Dis 2017; 12: 1401-1411.

6 Blasi F, Chalmers JD, Aliberti S. COPD and bronchiectasis: phenotype, endotype or co-morbidity? COPD J Chronic Obstr Pulm Dis 2014; 11: 603-604.

7 Clofent D, Álvarez A, Traversi L, et al. Comorbidities and mortality risk factors for patients with bronchiectasis. Expert Rev Respir Med 2021; 15: 623-634.

8 Polverino E, Goeminne PC, McDonnell MJ, et al. European Respiratory Society guidelines for the management of adult bronchiectasis. Eur Respir J 2017; 50: 1700629.

9 Quinti I, Soresina A, Spadaro G, et al. Long-term follow-up and outcome of a large cohort of patients with common variable immunodeficiency. J Clin Immunol 2007; 27: 308-316.

10 McDonnell MJ, Hunt EB, Ward C, et al. Current therapies for gastro-oesophageal reflux in the setting of chronic lung disease: state of the art review. ERJ Open Res 2020; 6: 00190-02019.

11 Chandrasekaran R, Mac Aogáin M, Chalmers JD, et al. Geographic variation in the aetiology, epidemiology and microbiology of bronchiectasis. BMC Pulm Med 2018; 18: 83.

12 Padilla-Galo A, Olveira C, Fernández de Rota-Garcia L, et al. Factors associated with bronchiectasis in patients with uncontrolled asthma; the NOPES score: a study in 398 patients. Respir Res 2018; 19: 43.

13 De la Rosa D, Martínez-Garcia M-A, Giron RM, et al. Clinical impact of chronic obstructive pulmonary disease on non-cystic fibrosis bronchiectasis. A study on 1,790 patients from the Spanish Bronchiectasis Historical Registry. PLoS One 2017; 12: e0177931.

14 Aksamit TR, O'Donnell AE, Barker A, et al. Adult patients with bronchiectasis: a first look at the US Bronchiectasis Research Registry. Chest 2017; 151: 982-992.

15 Pleasants R, Riley I, Mannino D. Defining and targeting health disparities in chronic obstructive pulmonary disease. Int J Chron Obstruct Pulmon Dis 2016; 11: 2475-2496.

16 Araújo D, Shteinberg M, Aliberti S, et al. Standardised classification of the aetiology of bronchiectasis using an objective algorithm. Eur Respir J 2017; 50: 1701289.

17 Polverino E, Dimakou K, Hurst J, et al. The overlap between bronchiectasis and chronic airway diseases: state of the art and future directions. Eur Respir J 2018; 52: 1800328.

18 Shi L, Wei F, Ma T, et al. Impact of radiographic bronchiectasis in COPD. Respir Care 2020; 65: 1561-1573.

19 Diaz AA, Young TP, Maselli DJ, et al. Quantitative CT measures of bronchiectasis in smokers. Chest 2017; 151: 1255-1262. 
20 Tan WC, Hague CJ, Leipsic J, et al. Findings on thoracic computed tomography scans and respiratory outcomes in persons with and without chronic obstructive pulmonary disease: a population-based cohort study. PLOS ONE 2016; 11: e0166745.

21 Monteagudo M, Rodríguez-Blanco T, Barrecheguren M, et al. Prevalence and incidence of bronchiectasis in Catalonia, Spain: a population-based study. Respir Med 2016; 121: 26-31.

22 Goeminne PC, Nawrot TS, Ruttens D, et al. Mortality in non-cystic fibrosis bronchiectasis: a prospective cohort analysis. Respir Med 2014; 108: 287-296.

23 Radovanovic D, Santus P, Blasi F, et al. A comprehensive approach to lung function in bronchiectasis. Respir Med 2018; 145: 120-129.

24 Martinez-García MA, Villa C, Dobarganes $\mathrm{Y}$, et al. RIBRON: el registro español informatizado de bronquiectasias. Caracterización de los primeros 1.912 pacientes. Arch Bronconeumol 2021; 57: 28-35.

25 Du Q, Jin J, Liu X, et al. Bronchiectasis as a comorbidity of chronic obstructive pulmonary disease: a systematic review and meta-analysis. PLOS ONE 2016; 11: e0150532.

26 Martínez-García MÁ, de la Rosa-Carrillo D, Soler-Cataluña JJ, et al. Bronchial infection and temporal evolution of bronchiectasis in patients with chronic obstructive pulmonary disease. Clin Infect Dis 2021; 72: 403-410.

27 Murphy MK, Black NA, Lamping DL, et al. Consensus development methods, and their use in clinical guideline development. Health Technol Assess (Rockv) 1998; 2: 1-88.

28 von der Gracht HA. Consensus measurement in Delphi studies. Review and implications for future quality assurance. Technol Forecast Soc Change 2012; 79: 1525-1536.

29 Hohmann E, Brand JC, Rossi MJ, et al. Expert opinion is necessary: Delphi panel methodology facilitates a scientific approach to consensus. Arthroscopy 2018; 34: 349-351.

30 McMillan SS, King M, Tully MP. How to use the nominal group and Delphi techniques. Int J Clin Pharm 2016; 38: 655-662.

31 Agusti A, Bel E, Thomas M, et al. Treatable traits: toward precision medicine of chronic airway diseases. Eur Respir J 2016; 47: 410-419.

32 McDonald VM, Fingleton J, Agusti A, et al. Treatable traits: a new paradigm for 21st century management of chronic airway diseases: Treatable Traits Down Under International Workshop report. Eur Respir J 2019; 53: 1802058.

33 Boaventura R, Sibila O, Agusti A, et al. Treatable traits in bronchiectasis. Eur Respir J 2018; 52: 1801269.

34 McDonnell MJ, Aliberti S, Goeminne PC, et al. Comorbidities and the risk of mortality in patients with bronchiectasis: an international multicentre cohort study. Lancet Respir Med 2016; 4: 969-979.

35 Aliberti S, Lonni S, Dore S, et al. Clinical phenotypes in adult patients with bronchiectasis. Eur Respir J 2016; 47: 1113-1122.

36 De Soyza A, McDonnell MJ, Goeminne PC, et al. Bronchiectasis rheumatoid overlap syndrome is an independent risk factor for mortality in patients with bronchiectasis: a multicenter cohort study. Chest 2017; 151: $1247-1254$

$37 \mathrm{Xu} \mathrm{B}$, Mao Y, Wan X, et al. Prognostic value of concomitant bronchiectasis in newly diagnosed diffuse panbronchiolitis patients on a maintenance therapy with macrolides. Can Respir J 2019; 2019: 1-7.

38 Crisafulli E, Guerrero M, lelpo A, et al. Impact of bronchiectasis on outcomes of hospitalized patients with acute exacerbation of chronic obstructive pulmonary disease: a propensity matched analysis. Sci Rep 2018; 8 : 9236.

39 Gatheral T, Kumar N, Sansom B, et al. COPD-related bronchiectasis; independent impact on disease course and outcomes. COPD J Chronic Obstr Pulm Dis 2014; 11: 605-614.

$40 \mathrm{Kim}$ YW, Jin KN, Heo EY, et al. The association between combined non-cystic fibrosis bronchiectasis and lung cancer in patients with chronic obstructive lung disease. Int J COPD 2015; 10: 873-879.

41 Hurst JR, Elborn JS, De Soyza A, et al. COPD-bronchiectasis overlap syndrome. Eur Respir J 2015; 45: 310-313.

42 Aliberti S, Polverino E, Chalmers JD, et al. The European multicentre Bronchiectasis Audit and Research Collaboration (EMBARC) ERS clinical research collaboration. Eur Respir J 2018; 52: 1802074.

43 Webb W, Müller NL, Naidich DP. Airways diseases. In: Webb WR, Müller NL, Naidich DP, eds. High-Resolution CT of the Lung, 3rd Edn. Philadelphia, Lippincott, Williams \& Wilkins, 2000; pp. 467-546.

44 Dou S, Zheng C, Ji X, et al. Co-existence of COPD and bronchiectasis: a risk factor for a high ratio of main pulmonary artery to aorta diameter (PA:A) from computed tomography in COPD patients. Int J COPD 2018; 13: $675-681$

45 Guan W, Gao Y, Xu G, et al. Characterization of lung function impairment in adults with bronchiectasis. PLoS ONE 2014; 9: e113373.

46 Dimakou K, Triantafillidou C, Toumbis M, et al. Non CF-bronchiectasis: aetiologic approach, clinical, radiological, microbiological and functional profile in 277 patients. Respir Med 2016; 116: 1-7.

47 Anwar GA, McDonnell MJ, Worthy SA, et al. Phenotyping adults with non-cystic fibrosis bronchiectasis: a prospective observational cohort study. Respir Med 2013; 107: 1001-1007.

48 Martinez-Garcia MÁ, Athanazio R, Gramblicka G, et al. Prognostic value of frequent exacerbations in bronchiectasis: the relationship with disease severity. Arch Bronconeumol 2018; 55: 81-87. 
Chalmers JD, Aliberti S, Filonenko A, et al. Characterization of the "Frequent Exacerbator Phenotype" in bronchiectasis. Am J Respir Crit Care Med 2018; 197: 1410-1420.

50 Capozzolo A, Carratù P, Dragonieri S, et al. Clinical and functional lung parameters associated with frequent exacerbator phenotype in subjects with severe COPD. Respir Care 2017; 62: 572-578.

51 Wedzicha JA, Brill SE, Allinson JP, et al. Mechanisms and impact of the frequent exacerbator phenotype in chronic obstructive pulmonary disease. BMC Med 2013; 11: 181.

52 Le Rouzic O, Roche N, Cortot AB, et al. Defining the "Frequent Exacerbator" phenotype in COPD. Chest 2018; 153: 1106-1115.

53 Pathak U, Gupta NC, Suri JC. Risk of COPD due to indoor air pollution from biomass cooking fuel: a systematic review and meta-analysis. Int J Environ Health Res 2020; 30: 75-88.

54 Colebatch HJ, Ng CK. Rate of increase in pulmonary distensibility in a longitudinal study of smokers. Thorax 1988; 43: 175-182.

55 Balcan B, Akan S, Ozsancak Ugurlu A, et al. Effects of biomass smoke on pulmonary functions: a case control study. Int J Chron Obstruct Pulmon Dis 2016; 11: 1615-1622.

56 Fang L, Gao P, Bao H, et al. Chronic obstructive pulmonary disease in China: a nationwide prevalence study. Lancet Respir Med 2018; 6: 421-430.

57 Martínez-García MÁ, Soler-Catalunã JJ, Sanz YD, et al. Factors associated with bronchiectasis in patients with COPD. Chest 2011; 140: 1130-1137.

$58 \mathrm{Jin} \mathrm{J}$, Yu W, Li S, et al. Factors associated with bronchiectasis in patients with moderate-severe chronic obstructive pulmonary disease. Medicine (Baltimore) 2016; 95: e4219.

59 Tufvesson E, Markstad H, Bozovic G, et al. Inflammation and chronic colonization of Haemophilus influenzae in sputum in COPD patients related to the degree of emphysema and bronchiectasis in high-resolution computed tomography. Int J COPD 2017; 12: 3211-3219.

60 Everaerts S, Lagrou K, Vermeersch K, et al. Aspergillus fumigatus detection and risk factors in patients with COPD-bronchiectasis overlap. Int J Mol Sci 2018; 19: 523.

61 de la Rosa Carrillo D, López-Campos JL, Alcázar Navarrete B, et al. Consensus document on the diagnosis and treatment of chronic bronchial infection in chronic obstructive pulmonary disease. Arch Bronconeumol (Engl Ed) 2020; 56: 651-664. 